\title{
On the symmetrical second order hyperbolic quaternions sequences
}

\author{
Sure Köme ${ }^{1}$ and Cahit Köme ${ }^{2}$ \\ ${ }^{1}$ Department of Mathematics, Nevşehir Hacı Bektaş Veli University, Turkey \\ e-mail: sure.kome@nevsehir.edu.tr \\ ${ }^{2}$ Department of Information Technology, Nevşehir Hacı Bektaş Veli University, Turkey \\ e-mail: cahit@nevsehir.edu.tr
}

Received: 4 September 2019 Revised: 26 November $2019 \quad$ Accepted: 20 April 2020

\begin{abstract}
The purpose of this study is to obtain a new generalized quaternions sequences by using hyperbolic functions with second order recurrence sequences. First of all, we define the symmetrical second order hyperbolic sine and the symmetrical second order hyperbolic cosine quaternions. Then, we investigate norms and some relations between these type of quaternions. We also obtain generating functions, Binet formulas, Catalan's identity, Cassini's identity and d'Ocagne's identity of second order hyperbolic quaternions sequences.
\end{abstract}

Keywords: Second order hyperbolic functions, Quaternions, Binet formula, Generating function.

2010 Mathematics Subject Classification: 11B37, 11R52, 05A15, $11 \mathrm{~B} 83$.

\section{Introduction}

Hyperbolic functions play an important role in many fields of mathematics, physics, geometry (Hyperbolic Lobatchevski's geometry) and cosmological researches (Four-dimensional Minkowki's world). Up until now, some researchers have studied the hyperbolic functions using number sequences [1,9,11-13]. For instance, Stakhov and Rozin illustrate some remarkable results for a new class of hyperbolic functions which unite the characteristics of the classical hyperbolic functions and the Fibonacci and Lucas series [11]. Falcon and Plaza introduced an extension of the classical Fibonacci hyperbolic functions, $k$-Fibonacci hyperbolic functions, and he 
presented several properties of these hyperbolic functions [2]. Stakhov and Rozin introduced the new continuous functions, which is an essential area of the Fibonacci numbers theory, for the Fibonacci and Lucas $p$-numbers with the help of Binet formulas [12]. By the help of the second order recurrence sequences, Koçer et al. proposed the symmetrical hyperbolic sine, $s U s(x)$, and symmetrical hyperbolic cosine hyperbolic functions, $c U s(x)$, as

$$
s U s(x)=\frac{\alpha^{x}-q^{x} \alpha^{-x}}{\sqrt{p^{2}+4 q}} \quad \text { and } \quad c U s(x)=\frac{\alpha^{x}+q^{x} \alpha^{-x}}{\sqrt{p^{2}+4 q}},
$$

where $p$ and $q$ are nonzero real numbers such that $p^{2}+4 q \neq 0$ and $\alpha$ is the root of the characteristic equation $x^{2}-p x-q$. They also showed that their study is generalization of some hyperbolic functions in the literature [9].

Another remarkable research topic is quaternions which are a number system that extends the complex numbers. In general, a quaternion $q$, which is member of a non-commutative division algebra, is defined by

$$
q=q_{0}+\mathbf{i} q_{1}+\mathbf{j} q_{2}+\mathbf{k} q_{3},
$$

where $q_{0}, q_{1}, q_{2}$ and $q_{3}$ are real numbers and $\mathbf{i}, \mathbf{j}$ and $\mathbf{k}$ satisfy the multiplication rules

$$
\mathbf{i}^{2}=\mathbf{j}^{2}=\mathbf{k}^{2}=-1, \quad \mathbf{i j}=-\mathbf{j i}=\mathbf{k}, \quad \mathbf{j k}=-\mathbf{k} \mathbf{j}=\mathbf{i}, \quad \mathbf{k i}=-\mathbf{i k}=\mathbf{j} .
$$

Quaternions arise in quantum mechanics, physics, mathematics, computer science and related areas. They have been studied by several authors in the recent years (see $[3-8,10])$. For example, Horadam, in [7], defined the Fibonacci quaternions. Motivated by Horadam's study, Halici, in [5], presented some basic properties of Fibonacci and Lucas quaternions. Flaut and Savin introduced the generalized Fibonacci-Lucas quaternions and they proved that the set of these elements is an order in the sense of ring theory of a quaternion algebra [4]. Köme et al demonstrated the modified generalized Fibonacci and Lucas quaternions and they presented the generating functions, the Binet formulas, matrix representations and some significant identities for these quaternions [10]. Using a different approach from our study, Catarino defined the hyperbolic $k$-Pell quaternions sequences and then she gave the generating functions, the Binet formulas and some identities for these quaternions sequences [1].

Motivating by the above cited works, in this paper, we define the symmetrical second order hyperbolic sine and the symmetrical second order hyperbolic cosine quaternions. After that, by using these quaternions and hyperbolic functions with second order recurrence sequences, we obtain the symmetrical second order hyperbolic quaternions sequences and we derive the generating functions, the Binet formula and some important properties of these quaternions sequences.

\section{Main results}

Definition 1. For any $p, q \in \mathbb{R}^{+}$and $x \in \mathbb{R}$, the symmetrical second order hyperbolic sine and the symmetrical second order hyperbolic cosine quaternions are defined by

$$
s U_{s}(x) Q=s U_{s}(x)+s U_{s}(x+1) i_{1}+s U_{s}(x+2) i_{2}+s U_{s}(x+3) i_{3}
$$


and

$$
c U_{s}(x) Q=c U_{s}(x)+c U_{s}(x+1) i_{1}+c U_{s}(x+2) i_{2}+c U_{s}(x+3) i_{3},
$$

where $s U_{s}(x)$ and $c U_{s}(x)$ are the symmetrical second order hyperbolic functions which are defined in the Eq. (1).

The following theorem shows the relation between the symmetrical second order hyperbolic sine and the symmetrical second order hyperbolic cosine quaternions.

Theorem 2.1. For any $x \in \mathbb{R}$, the following relations hold:

$$
\begin{aligned}
& s U_{s}(x+2) Q=p c U_{s}(x+1) Q+q s U_{s}(x) Q \\
& c U_{s}(x+2) Q=p s U_{s}(x+1) Q+q c U_{s}(x) Q .
\end{aligned}
$$

Proof. By using the equations (4), (5) and Proposition 1 in [9], we have

$$
\begin{aligned}
p c U_{s}(x+1) Q+q s U_{s}(x) Q= & p\left(c U_{s}(x+1)+c U_{s}(x+2) i_{1}+c U_{s}(x+3) i_{2}+c U_{s}(x+4) i_{3}\right) \\
& +q\left(s U_{s}(x)+s U_{s}(x+1) i_{1}+s U_{s}(x+2) i_{2}+s U_{s}(x+3) i_{3}\right) \\
= & \left(p c U_{s}(x+1)+q s U_{s}(x)\right)+\left(p c U_{s}(x+2)+q s U_{s}(x+1)\right) i_{1} \\
& +\left(p c U_{s}(x+3)+q s U_{s}(x+2)\right) i_{2}+\left(p c U_{s}(x+4)+q s U_{s}(x+3)\right) i_{3} \\
= & s U_{s}(x+2)+s U_{s}(x+3) i_{1}+s U_{s}(x+4) i_{2}+s U_{s}(x+5) i_{3} \\
= & s U_{s}(x+2) Q .
\end{aligned}
$$

Analogously, we can prove the second relation. So we omit the proof.

Theorem 2.2. For any $x \in \mathbb{R}$, the norm identities of the symmetrical second order hyperbolic sine and the symmetrical second order hyperbolic cosine quaternions are as follows:

$$
\begin{aligned}
& \left\|s U_{s}(x) Q\right\|^{2}=\frac{\alpha^{2 x}\left(\alpha^{2}+1\right)\left(\alpha^{4}+1\right)+q^{2 x} \alpha^{-2 x-6}\left(q^{2}+\alpha^{2}\right)\left(q^{4}+\alpha^{4}\right)-2 q^{x}(q+1)\left(q^{2}+1\right)}{\left(\alpha+q \alpha^{-1}\right)^{2}} \\
& \left\|c U_{s}(x) Q\right\|^{2}=\frac{\alpha^{2 x}\left(\alpha^{2}+1\right)\left(\alpha^{4}+1\right)+q^{2 x} \alpha^{-2 x-6}\left(q^{2}+\alpha^{2}\right)\left(q^{4}+\alpha^{4}\right)+2 q^{x}(q+1)\left(q^{2}+1\right)}{\left(\alpha+q \alpha^{-1}\right)^{2}} .
\end{aligned}
$$

Proof. By using the equations in (1), we obtain

$$
\begin{aligned}
\left\|s U_{s}(x) Q\right\|^{2}= & \left(s U_{s}(x)\right)^{2}+\left(s U_{s}(x+1)\right)^{2}+\left(s U_{s}(x+2)\right)^{2}+\left(s U_{s}(x+3)\right)^{2} \\
= & \left(\frac{\alpha^{x}-q^{x} \alpha^{-x}}{\alpha+q \alpha^{-1}}\right)^{2}+\left(\frac{\alpha^{x+1}-q^{x+1} \alpha^{-x-1}}{\alpha+q \alpha^{-1}}\right)^{2}+\left(\frac{\alpha^{x+2}-q^{x+2} \alpha^{-x-2}}{\alpha+q \alpha^{-1}}\right)^{2} \\
& \quad+\left(\frac{\alpha^{x+3}-q^{x+3} \alpha^{-x-3}}{\alpha+q \alpha^{-1}}\right)^{2} \\
= & \frac{\alpha^{2 x}}{\left(\alpha+q \alpha^{-1}\right)^{2}}\left(1+\alpha^{2}+\alpha^{4}+\alpha^{6}\right)+\frac{q^{2 x} \alpha^{-2 x}}{\left(\alpha+q \alpha^{-1}\right)^{2}}\left(1+\left(\frac{q}{\alpha}\right)^{2}+\left(\frac{q}{\alpha}\right)^{4}+\left(\frac{q}{\alpha}\right)^{6}\right) \\
& \quad-\frac{2 q^{x}}{\left(\alpha+q \alpha^{-1}\right)^{2}}\left(1+q+q^{2}+q^{3}\right)
\end{aligned}
$$




$$
\begin{aligned}
& =\frac{\alpha^{2 x}}{\left(\alpha+q \alpha^{-1}\right)^{2}}\left(\frac{\alpha^{8}-1}{\alpha^{2}-1}\right)+\frac{q^{2 x} \alpha^{-2 x}}{\left(\alpha+q \alpha^{-1}\right)^{2}}\left(\frac{\left(\frac{q}{\alpha}\right)^{8}-1}{\left(\frac{q}{\alpha}\right)^{2}-1}\right)-\frac{2 q^{x}}{\left(\alpha+q \alpha^{-1}\right)^{2}}\left(\frac{q^{4}-1}{q-1}\right) \\
& =\frac{\alpha^{2 x}\left(\alpha^{2}+1\right)\left(\alpha^{4}+1\right)+q^{2 x} \alpha^{-2 x-6}\left(q^{2}+\alpha^{2}\right)\left(q^{4}+\alpha^{4}\right)-2 q^{x}(q+1)\left(q^{2}+1\right)}{\left(\alpha+q \alpha^{-1}\right)^{2}} .
\end{aligned}
$$

Similarly, we obtain

$$
\left\|c U_{s}(x) Q\right\|^{2}=\frac{\alpha^{2 x}\left(\alpha^{2}+1\right)\left(\alpha^{4}+1\right)+q^{2 x} \alpha^{-2 x-6}\left(q^{2}+\alpha^{2}\right)\left(q^{4}+\alpha^{4}\right)+2 q^{x}(q+1)\left(q^{2}+1\right)}{\left(\alpha+q \alpha^{-1}\right)^{2}}
$$

which completes the proof.

Theorem 2.3. For any $x \in \mathbb{R}$, the symmetrical second order hyperbolic sine and the symmetrical second order hyperbolic cosine quaternions satisfy the following relations:

$$
s U_{s}(x+2) Q=\chi(q) s U_{s}(x) Q-s U_{s}(x-2) Q
$$

and

$$
c U_{s}(x+2) Q=\chi(q) c U_{s}(x) Q-c U_{s}(x-2) Q,
$$

where the transformation function $\chi(q)=\lambda(q)^{2} \alpha^{-2}+\lambda(q)^{-2} \alpha^{2}$ and where

$$
\lambda(q)= \begin{cases}1, & \chi(q) \alpha^{x} \\ q, & \chi(q) \alpha^{-x}\end{cases}
$$

Proof. By using the equations (4) and (5), we have

$$
\begin{aligned}
& s U_{s}(x+2) Q+s U_{s}(x-2) Q \\
& =\left(s U_{s}(x+2)+s U_{s}(x-2)\right)+\left(s U_{s}(x+3)+s U_{s}(x-1)\right) i_{1} \\
& +\left(s U_{s}(x+4)+s U_{s}(x)\right) i_{2}+\left(s U_{s}(x+5)+s U_{s}(x+1)\right) i_{3} \\
& =\frac{\alpha^{x}\left(\alpha^{2}+\alpha^{-2}\right)-q^{x} \alpha^{-x}\left(q^{2} \alpha^{-2}+q^{-2} \alpha^{2}\right)}{\alpha+q \alpha^{-1}} \\
& +\left(\frac{\alpha^{x+1}\left(\alpha^{2}+\alpha^{-2}\right)-q^{x+1} \alpha^{-x-1}\left(q^{2} \alpha^{-2}+q^{-2} \alpha^{2}\right)}{\alpha+q \alpha^{-1}}\right) i_{1} \\
& +\left(\frac{\alpha^{x+2}\left(\alpha^{2}+\alpha^{-2}\right)-q^{x+2} \alpha^{-x-2}\left(q^{2} \alpha^{-2}+q^{-2} \alpha^{2}\right)}{\alpha+q \alpha^{-1}}\right) i_{2} \\
& +\left(\frac{\alpha^{x+3}\left(\alpha^{2}+\alpha^{-2}\right)-q^{x+3} \alpha^{-x-3}\left(q^{2} \alpha^{-2}+q^{-2} \alpha^{2}\right)}{\alpha+q \alpha^{-1}}\right) i_{3} \\
& =\chi(q)\left(s U_{s}(x)+s U_{s}(x+1) i_{1}+s U_{s}(x+2) i_{2}+s U_{s}(x+3) i_{3}\right) \\
& =\chi(q) s U_{s}(x) Q \text {. }
\end{aligned}
$$


In the same way, we get

$$
c U_{s}(x+2) Q+c U_{s}(x-2) Q=\chi(q) c U_{s}(x) Q .
$$

Hence the proof is complete.

Now, we give the generating functions for the symmetrical second order hyperbolic quaternions sequences.

Theorem 2.4. For any $x \in \mathbb{R}$, the generating functions for the symmetrical second order hyperbolic quaternions sequences are as follows:

$$
G_{s}(x, t)=\frac{s U_{s}(x) Q+s U_{s}(x+1) Q t-s U_{s}(x-2) Q t^{2}-s U_{s}(x+1) Q t^{3}}{1-\chi(q) t^{2}+t^{4}}
$$

and

$$
G_{c}(x, t)=\frac{c U_{s}(x) Q+c U_{s}(x+1) Q t-c U_{s}(x-2) Q t^{2}-c U_{s}(x+1) Q t^{3}}{1-\chi(q) t^{2}+t^{4}} .
$$

Proof. We use the formal power series to find the generating function of the symmetrical second order hyperbolic quaternions sequences. Now, we define

$$
\begin{gathered}
G_{s}(x, t)=\sum_{n=0}^{\infty} s U_{s}(x+n) Q t^{n}=s U_{s}(x) Q+s U_{s}(x+1) Q t \\
+s U_{s}(x+2) Q t^{2}+s U_{s}(x+3) Q t^{3}+\sum_{n=4}^{\infty} s U_{s}(x+n) Q t^{n} \\
-\chi(q) G_{s}(x, t) t^{2}=-\chi(q) \sum_{n=0}^{\infty} s U_{s}(x+n) Q t^{n+2}=-\chi(q) \sum_{n=2}^{\infty} s U_{s}(x+n-2) Q t^{n} \\
=-\chi(q)\left(s U_{s}(x) Q t^{2}+s U_{s}(x+1) Q t^{3}+\sum_{n=4}^{\infty} s U_{s}(x+n-2) Q t^{n}\right) \\
t^{4} G_{s}(x, t)=\sum_{n=0}^{\infty} s U_{s}(x+n) Q t^{n+4}=\sum_{n=4}^{\infty} s U_{s}(x+n-4) Q t^{n} .
\end{gathered}
$$

By using above expressions, we have

$$
\begin{aligned}
& \left(1-\chi(q) t^{2}+t^{4}\right) G_{s}(x, t) \\
= & s U_{s}(x) Q+s U_{s}(x+1) Q t+s U_{s}(x+2) Q t^{2}+s U_{s}(x+3) Q t^{3} \\
& -\chi(q) s U_{s}(x) Q t^{2}-\chi(q) s U_{s}(x+1) Q t^{3} \\
& +\sum_{n=4}^{\infty}\left(s U_{s}(x+n) Q-\chi(q) s U_{s}(x+n-2) Q+s U_{s}(x+n-4) Q\right) t^{n} \\
= & s U_{s}(x) Q+s U_{s}(x+1) Q t-s U_{s}(x-2) Q t^{2}-s U_{s}(x-1) Q t^{3} \\
& +\sum_{n=4}^{\infty}(\underbrace{s U_{s}(x+n) Q-\chi(q) s U_{s}(x+n-2) Q+s U_{s}(x+n-4) Q}_{0}) t^{n} .
\end{aligned}
$$


Hence, we have

$$
G_{s}(x, t)=\frac{s U_{s}(x) Q+s U_{s}(x+1) Q t-s U_{s}(x-2) Q t^{2}-s U_{s}(x-1) Q t^{3}}{1-\chi(q) t^{2}+t^{4}} .
$$

By doing similar operations, we get

$$
G_{c}(x, t)=\frac{c U_{s}(x) Q+c U_{s}(x+1) Q t-c U_{s}(x-2) Q t^{2}-c U_{s}(x-1) Q t^{3}}{1-\chi(q) t^{2}+t^{4}} .
$$

Therefore the proof is complete.

Theorem 2.5. The Binet formulas for the symmetrical second order quaternions sequences are as follows:

$$
s U_{s}(x+n) Q=\frac{A \alpha^{x+n}-B q^{x+n} \alpha^{-x-n}}{\alpha+q \alpha^{-1}}
$$

and

$$
c U_{s}(x+n) Q=\frac{A \alpha^{x+n}+B q^{x+n} \alpha^{-x-n}}{\alpha+q \alpha^{-1}},
$$

where $A=1+\alpha i_{1}+\alpha^{2} i_{2}+\alpha^{3} i_{3}$ and $B=1+q \alpha^{-1} i_{1}+q^{2} \alpha^{-2} i_{2}+q^{3} \alpha^{-3} i_{3}$.

Proof.

$$
\begin{aligned}
s U_{s}(x+n) Q= & s U_{s}(x+n)+s U_{s}(x+n+1) i_{1}+s U_{s}(x+n+2) i_{2}+s U_{s}(x+n+3) i_{3} \\
= & \frac{\alpha^{x+n}-q^{x+n} \alpha^{-x-n}}{\alpha+q \alpha^{-1}}+\frac{\alpha^{x+n+1}-q^{x+n+1} \alpha^{-x-n-1}}{\alpha+q \alpha^{-1}} i_{1} \\
& \quad+\frac{\alpha^{x+n+2}-q^{x+n+2} \alpha^{-x-n-2}}{\alpha+q \alpha^{-1}} i_{2}+\frac{\alpha^{x+n+3}-q^{x+n+3} \alpha^{-x-n-3}}{\alpha+q \alpha^{-1}} i_{3} \\
= & \frac{\alpha^{x+n}}{\alpha+q \alpha^{-1}}\left(1+\alpha i_{1}+\alpha^{2} i_{2}+\alpha^{3} i_{3}\right) \\
& -\frac{q^{x+n} \alpha^{-x-n}}{\alpha+q \alpha^{-1}}\left(1+q \alpha^{-1} i_{1}+q^{2} \alpha^{-2} i_{2}+q^{3} \alpha^{-3} i_{3}\right) \\
= & \frac{A \alpha^{x+n}-B q^{x+n} \alpha^{-x-n}}{\alpha+q \alpha^{-1}} .
\end{aligned}
$$

In a similar way, we get

$$
c U_{s}(x+n) Q=\frac{A \alpha^{x+n}+B q^{x+n} \alpha^{-x-n}}{\alpha+q \alpha^{-1}} .
$$

Hence the proof is complete.

It is not difficult to see that the symmetrical second order hyperbolic quaternions sequences can be reduced into several quaternions sequences for the special cases of $p$ and $q$. For example,

- If we get $p=k$ and $q=1$, we have $k$-Fibonacci hyperbolic quaternions sequences; 
- If we get $p=k$ and $q=2$, we have $k$-Jacobsthal hyperbolic quaternions sequences;

- If we get $p=2$ and $q=k$, we have $k$-Pell hyperbolic quaternions sequences;

- If we get $p=2$ and $q=1$, we have Pell hyperbolic quaternions sequences;

- If we get $p=1$ and $q=2$, we have Jacobsthal hyperbolic quaternions sequences;

- If we get $p=1$ and $q=1$, we have Fibonacci hyperbolic quaternions sequences.

Now, we give the well-known identities such as Catalan's identity, Cassini's identity and d'Ocagne's identity for the symmetrical second order quaternions sequences.

Theorem 2.6. For nonnegative integers $n$ and $r$, such that $r \leq n$, the Catalan identities for the symmetrical second order quaternions sequences are as follows:

$$
\begin{aligned}
& s U_{s}(x+n+r) Q s U_{s}(x+n-r) Q-\left(s U_{s}(x+n) Q\right)^{2} \\
& \quad=\frac{1}{\left(\alpha+q \alpha^{-1}\right)^{2}}\left(A B\left(q^{x+n}-q^{x+n-r} \alpha^{2 r}\right)+B A\left(q^{x+n}-q^{x+n+r} \alpha^{-2 r}\right)\right)
\end{aligned}
$$

and

$$
\begin{aligned}
& c U_{s}(x+n+r) Q c U_{s}(x+n-r) Q-\left(c U_{s}(x+n) Q\right)^{2} \\
& \quad=\frac{1}{\left(\alpha+q \alpha^{-1}\right)^{2}}\left(-A B\left(q^{x+n}-q^{x+n-r} \alpha^{2 r}\right)-B A\left(q^{x+n}-q^{x+n+r} \alpha^{-2 r}\right)\right),
\end{aligned}
$$

where $A=1+\alpha i_{1}+\alpha^{2} i_{2}+\alpha^{3} i_{3}$ and $B=1+q \alpha^{-1} i_{1}+q^{2} \alpha^{-2} i_{2}+q^{3} \alpha^{-3} i_{3}$.

Proof. By using the Binet formula of the symmetrical second order quaternions sequences in Theorem 2.5, we get

$$
\begin{aligned}
s U_{s} & (x+n+r) Q s U_{s}(x+n-r) Q-\left(s U_{s}(x+n) Q\right)^{2} \\
& =\left(\frac{A \alpha^{x+n+r}-B q^{x+n+r} \alpha^{-x-n-r}}{\alpha+q \alpha^{-1}}\right)\left(\frac{A \alpha^{x+n-r}-B q^{x+n-r} \alpha^{-x-n+r}}{\alpha+q \alpha^{-1}}\right) \\
& -\left(\frac{A \alpha^{x+n}-B q^{x+n} \alpha^{-x-n}}{\alpha+q \alpha^{-1}}\right)^{2} \\
& =\frac{-A B q^{x+n-r} \alpha^{2 r}-B A q^{x+n+r} \alpha^{-2 r}+A B q^{x+n}+B A q^{x+n}}{\left(\alpha+q \alpha^{-1}\right)^{2}} \\
& =\frac{1}{\left(\alpha+q \alpha^{-1}\right)^{2}}\left(A B\left(q^{x+n}-q^{x+n-r} \alpha^{2 r}\right)+B A\left(q^{x+n}-q^{x+n+r} \alpha^{-2 r}\right)\right) .
\end{aligned}
$$

Similarly, we get

$$
\begin{aligned}
& c U_{s}(x+n+r) Q c U_{s}(x+n-r) Q-\left(c U_{s}(x+n) Q\right)^{2} \\
& \quad=\frac{1}{\left(\alpha+q \alpha^{-1}\right)^{2}}\left(-A B\left(q^{x+n}-q^{x+n-r} \alpha^{2 r}\right)-B A\left(q^{x+n}-q^{x+n+r} \alpha^{-2 r}\right)\right) .
\end{aligned}
$$

Hence we prove the theorem. 
Corollary 2.6.1. For $r=1$, we obtain the Cassini's identity for the symmetrical second order quaternions sequences as

$$
\begin{aligned}
& s U_{s}(x+n+1) Q s U_{s}(x+n-1) Q-\left(s U_{s}(x+n) Q\right)^{2} \\
& \quad=\frac{1}{\left(\alpha+q \alpha^{-1}\right)^{2}}\left(A B\left(q^{x+n}-q^{x+n-1} \alpha^{2}\right)+B A\left(q^{x+n}-q^{x+n+1} \alpha^{-2}\right)\right)
\end{aligned}
$$

and

$$
\begin{aligned}
& c U_{s}(x+n+1) Q c U_{s}(x+n-1) Q-\left(c U_{s}(x+n) Q\right)^{2} \\
& \quad=\frac{1}{\left(\alpha+q \alpha^{-1}\right)^{2}}\left(-A B\left(q^{x+n}-q^{x+n-1} \alpha^{2}\right)-B A\left(q^{x+n}-q^{x+n+1} \alpha^{-2}\right)\right),
\end{aligned}
$$

respectively.

Theorem 2.7 (d'Ocagne's identity). For the symmetrical second order quaternions sequences, the following identities hold:

$$
\begin{gathered}
s U_{s}(x+m) Q s U_{s}(x+n+1) Q-s U_{s}(x+m+1) Q s U_{s}(x+n) Q \\
\quad=\frac{\alpha-q \alpha^{-1}}{\left(\alpha+q \alpha^{-1}\right)^{2}}\left(A B q^{x+n} \alpha^{m-n}-B A q^{x+m} \alpha^{n-m}\right)
\end{gathered}
$$

and

$$
\begin{gathered}
c U_{s}(x+m) Q c U_{s}(x+n+1) Q-c U_{s}(x+m+1) Q c U_{s}(x+n) Q \\
=\frac{\alpha-q \alpha^{-1}}{\left(\alpha+q \alpha^{-1}\right)^{2}}\left(-A B q^{x+n} \alpha^{m-n}+B A q^{x+m} \alpha^{n-m}\right),
\end{gathered}
$$

where $A=1+\alpha i_{1}+\alpha^{2} i_{2}+\alpha^{3} i_{3}$ and $B=1+q \alpha^{-1} i_{1}+q^{2} \alpha^{-2} i_{2}+q^{3} \alpha^{-3} i_{3}$.

Proof. By using the Binet formula of the symmetrical second order quaternions sequences in Theorem 2.5, we get

$$
\begin{aligned}
s U_{s}( & x+m) Q s U_{s}(x+n+1) Q-s U_{s}(x+m+1) Q s U_{s}(x+n) Q \\
= & \left(\frac{A \alpha^{x+m}-B q^{x+m} \alpha^{-x-m}}{\alpha+q \alpha^{-1}}\right)\left(\frac{A \alpha^{x+n+1}-B q^{x+n+1} \alpha^{-x-n-1}}{\alpha+q \alpha^{-1}}\right) \\
& \quad-\left(\frac{A \alpha^{x+m+1}-B q^{x+m+1} \alpha^{-x-m-1}}{\alpha+q \alpha^{-1}}\right)\left(\frac{A \alpha^{x+n}-B q^{x+n} \alpha^{-x-n}}{\alpha+q \alpha^{-1}}\right) \\
= & \frac{A B q^{x+n} \alpha^{m-n+1}+B A q^{x+m+1} \alpha^{n-m-1}-A B q^{x+n+1} \alpha^{m-n-1}-B A q^{x+m} \alpha^{n-m+1}}{\left(\alpha+q \alpha^{-1}\right)^{2}} \\
= & \frac{A B q^{x+n} \alpha^{m-n}\left(\alpha-q \alpha^{-1}\right)+B A q^{x+m} \alpha^{n-m}\left(q \alpha^{-1}-\alpha\right)}{\left(\alpha+q \alpha^{-1}\right)^{2}} \\
= & \frac{\alpha-q \alpha^{-1}}{\left(\alpha+q \alpha^{-1}\right)^{2}}\left(A B q^{x+n} \alpha^{m-n}-B A q^{x+m} \alpha^{n-m}\right) .
\end{aligned}
$$


Similarly, we get

$$
\begin{gathered}
c U_{s}(x+m) Q c U_{s}(x+n+1) Q-c U_{s}(x+m+1) Q c U_{s}(x+n) Q \\
=\frac{\alpha-q \alpha^{-1}}{\left(\alpha+q \alpha^{-1}\right)^{2}}\left(-A B q^{x+n} \alpha^{m-n}+B A q^{x+m} \alpha^{n-m}\right),
\end{gathered}
$$

which proves the theorem.

\section{Conclusion}

This study presents the symmetrical second order quaternions sequences and their norms, generating functions and many properties of these quaternions sequences. Moreover, for the special cases of $p$ and $q$, we obtain several new quaternions sequences which have not been defined before. We also obtain generating functions, Binet formulas, Catalan's identity, Cassini's identity and d'Ocagne's identity of second order hyperbolic quaternions sequences. Since this study includes some new generalized results for the quaternions sequences, it contributes to the literature by providing essential information on the generalization of the quaternions sequences.

\section{References}

[1] Catarino, P. (2018). On hyperbolic $k$-Pell quaternions sequences, In Annales Mathematicae et Informaticae, 49, 61-73.

[2] Falcon, S., \& Plaza, A. (2008). The $k$-Fibonacci hyperbolic functions, Chaos, Solitons \& Fractals, 38 (2), 409-420.

[3] Flaut, C., \& Shpakivskyi, V. (2013). Real matrix representations for the complex quaternions, Advances in Applied Clifford Algebras, 23 (3), 657-671.

[4] Flaut, C., \& Savin, D. (2015). Quaternion algebras and generalized Fibonacci-Lucas quaternions, Advances in Applied Clifford Algebras, 25 (4), 853-862.

[5] Halici, S. (2012). On Fibonacci quaternions, Advances in Applied Clifford Algebras, 22 (2), 321-327.

[6] Hamilton, W. R. (1866). Elements of Quaternions, Longmans, Green \& Company.

[7] Horadam, A. F. (1963). Complex Fibonacci numbers and Fibonacci quaternions, The American Mathematical Monthly, 70 (3), 289-291.

[8] Horadam, A. F. (1993). Quaternion recurrence relations, Ulam Quarterly, 2 (2), 23-33.

[9] Kocer, E. G., Tuglu, N., \& Stakhov, A. (2008). Hyperbolic functions with second order recurrence sequences, Ars Combinatoria, 88, 65-81. 
[10] Köme, S., Köme, C., \& Yazlik, Y. (2019). Modified generalized Fibonacci and Lucas quaternions, Journal of Science and Arts, 19 (1), 49-60.

[11] Stakhov, A., \& Rozin, B. (2005). On a new class of hyperbolic functions, Chaos, Solitons \& Fractals, 23 (2), 379-389.

[12] Stakhov, A., \& Rozin, B. (2006). The continuous functions for the Fibonacci and Lucas p-numbers, Chaos, Solitons \& Fractals, 28 (4), 1014-1025.

[13] Stakhov, A., \& Rozin, B. (2005). The golden shofar, Chaos, Solitons \& Fractals, 26 (3), 677-684. 